\title{
Comparison of antiplatelet treatment in patients with clopidogrel nonresponders with or without carriage of $\mathrm{CYP}_{2} \mathrm{C}_{19}$ polymorphism
}

Yun Myoung $\mathrm{Ko}^{1}$, Jeong Kyung Kim² ${ }^{2}$ Jeong Hee Kim², Sang-Ho Park ${ }^{3}$, and Rak Kyeong Choi ${ }^{4}$

${ }^{1}$ Department of Internal Medicine, ${ }^{2}$ Cardiovascular Center, Department of Internal Medicine, Sun Hospital, Daejeon; ${ }^{3}$ Department of Internal Medicine, Soonchunhyang University Hospital Cheonan, Cheonan; ${ }^{4}$ Division of Cardiovascular, Department of Internal Medicine, Mediplex Sejong Hospital, Incheon, Korea
Received: October 31, 2017

Revised : December 8, 2017

Accepted: January 8, 2018

\section{Correspondence to} Jeong Kyung Kim, M.D. Cardiovascular Center, Department of Internal Medicine, Sun Hospital, 29 Mokjung-ro, Jung-gu, Daejeon 34811, Korea

Tel: +82-42-220-8744

Fax: +82-42-252-5505

E-mail:mdzoa@daum.net
Background/Aims: Several interventions exist for overcoming high platelet reactivity (HPR) on clopidogrel therapy. The goal of this study was to identify strategies that improve inhibition of platelet reactivity in clopidogrel nonresponders with or without loss of function $\mathrm{CrP}_{2} \mathrm{C}_{1} 9$ genotypes, resulting in platelet reactivity similar to that in responders.

Methods: A total of 376 patients with stenting for coronary artery disease underwent platelet function testing in three centers. Blinded platelet function tests were performed after $75 \mathrm{mg}$ daily clopidogrel treatment for 28 days. In total, 183 nonresponders were genotyped, were randomized to four treatment groups with each treatment lasting approximately 28 days, and underwent repeated measurements of platelet reactivity after treatment.

Results: With $75 \mathrm{mg}$ of daily clopidogrel, nonresponders had significantly higher HPR than did responders (multiple electrode aggregometry [MEA, arbitrary platelet aggregation unit]: mean, 71.4; 95\% confidence intervals [CI], 68.6 to 74.3; and mean, 27.5 ; 95\% CI, 26.0 to 28.9 , respectively; $p<0.001$ ). Ticagrelor or ticlopidine treatment in nonresponders resulted in platelet reactivity similar to that in responders in intermediate metabolizers (mean, 24.0; 95\% CI, 19.6 to 28.4 ; $p>0.05$; and mean, 30.0; 95\% CI, 24.7 to 37.5; $p>0.05$, respectively) and poor metabolizers (mean, 23.2; 95\% CI, 18.0 to 28.3 ; $p>0.05$; and mean, 30.3; 95\% CI, 24.5 to 6.0 ; $p$ > 0.05 , respectively). However, in extensive metabolizers, only ticagrelor treatment showed platelet reactivity similar to that in responders (mean, 26.1; 95\% CI, 24.1 to $28.0 ; p>0.05$ ).

Conclusions: Among clopidogrel nonresponders with cardiovascular disease on $75 \mathrm{mg}$ daily clopidogrel, ticagrelor resulted in a comparable degree of platelet inhibition in all nonresponders compared with $150 \mathrm{mg}$ daily clopidogrel or triple therapy with clopidogrel and cilostazol, irrespective of phenotype.

Keywords: Stable cardiovascular disease; Clopidogrel; Cytochrome 2C19 polymorphism 


\section{INTRODUCTION}

Clopidogrel, an oral thienopyridine antiplatelet drug, has been reported to be effective when combined with aspirin in preventing atherothrombotic events in patients with acute coronary syndrome (ACS), particular when percutaneous coronary intervention (PCI) is performed [1].

Dual antiplatelet therapy with aspirin and standard $75 \mathrm{mg}$ maintenance doses of clopidogrel has become the cornerstone of medical regimens for prevention of ischemic events in patients undergoing PCI with stent placement. However, variability in pharmacodynamic response to clopidogrel is well-recognized, and patients with high platelet reactivity (HPR) have an increased risk of adverse cardiovascular events [2].

Clopidogrel biotransformation is mainly mediated by the hepatic cytochrome P450 (CYP) enzymes. Recent studies have shown that both heterozygous and homozygous loss of function (LOF) allele carriers have a marked decrease in platelet response with the standard $75 \mathrm{mg}$ maintenance dose of clopidogrel [3,4], and higher rates of adverse cardiovascular events compared with non-carriers [5].
Guidance is needed regarding optimal treatment strategies in patients with high clopidogrel on-treatment platelet reactivity with or without $\mathrm{CrP}_{2} \mathrm{C}_{19} \mathrm{LOF}$ polymorphism.

Although various $\mathrm{P}_{2} \mathrm{Y} 12$ inhibitors that might be unaffected by the $\mathrm{CrP}_{2} \mathrm{C}_{19}$ genotype are available, these medications may be expensive and not globally accessible, particularly in Asia. In contrast, clopidogrel, cilostazol, and ticlopidine are widely available.

Therefore, we conducted repeated measurements of platelet reactivity in patients with HPR with or without

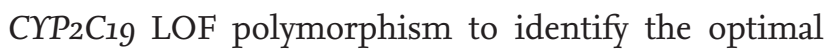
treatment strategy among $150 \mathrm{mg}$ maintenance doses of clopidogrel, $75 \mathrm{mg}$ daily clopidogrel, $100 \mathrm{mg}$ twice-daily cilostazol, $250 \mathrm{mg}$ twice-daily ticlopidine, and $90 \mathrm{mg}$ twice-daily ticagrelor.

\section{METHODS}

Patients were considered eligible to be enrolled if they had coronary artery disease with stenting and were on daily $75 \mathrm{mg}$ clopidogrel. To be eligible, patients needed to have an indication for the use of clopidogrel (a

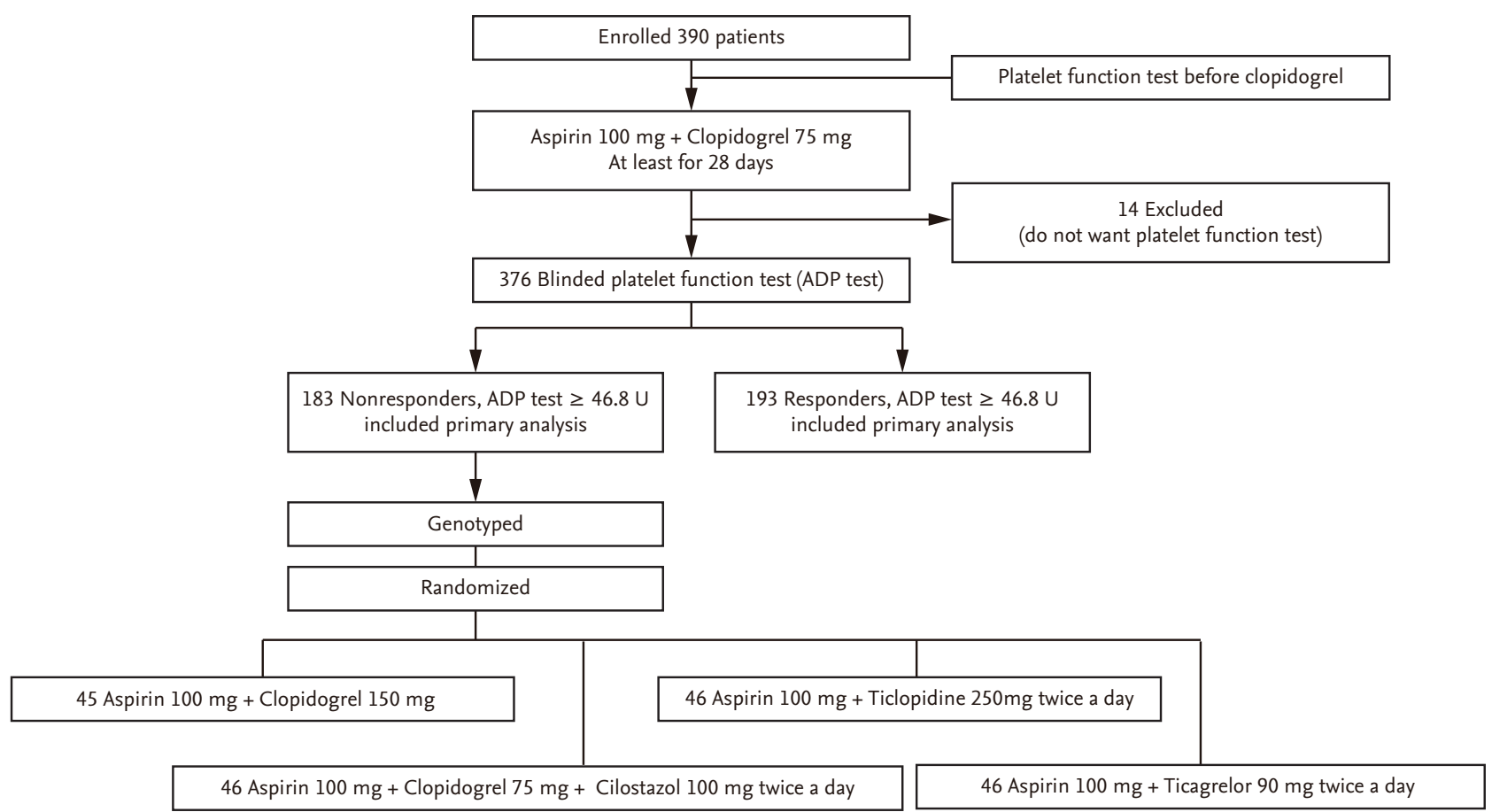

Figure 1. Patient flow diagram. ADP, adenosine diphosphate. 
myocardial infarction and/or PCI $\geq 4$ weeks and $\leq 6$ months prior to enrollment) and had to have a clinically stable state. In total, 390 patients from three sites were screened. A patient enrollment flow diagram is shown in Fig. 1. All patients were requested to continue daily $100 \mathrm{mg}$ aspirin during the study. Key exclusion criteria were the use of anticoagulants or proton pump inhibitors, current smoking, prior stent thrombosis, heightened risk of bleeding, end-stage renal or hepatic disease, or a procedure or hospitalization scheduled for the 12 subsequent weeks.

\section{Study protocol}

Baseline clinical evaluation was performed at the time of enrollment, and each patient underwent blood sampling for blinded platelet function testing. Blood sampling was done 2 hours after but not more than 3 hours after the last drug dose.

Platelet function was assessed using multiple electrode aggregometry (MEA) (Multiplate analyzer, Dynabyte, Munich, Germany). This system detects changes in electrical impedance due to adhesion and aggregation of platelets on two independent electrode-set surfaces in the test cuvette. A 1:2 dilution of whole blood treated with the anticoagulant hirudin and $0.9 \% \mathrm{NaCl}$ was stirred at $37^{\circ} \mathrm{C}$ for 3 minutes in the test cuvettes, $6.4 \mu \mathrm{M}$ adenosine diphosphate (ADP) and $9.4 \mathrm{nM}$ prostaglandin E1 were added, and increase in electrical impedance was recorded continuously for 6 minutes. Mean values of two independent determinations were expressed as the area under the curve (AUC) of the aggregation tracing. Impedance measured with MEA was transformed to arbitrary aggregation units $(\mathrm{U})$ which were plotted against time (U. min). We reported AUC in units (U). Good reproducibility of MEA measurements has been reported previously (<6\% variability) [6]. All measurements were obtained by laboratory personnel who were unaware of results of phenotyping or clinical outcomes of patients. Laboratory imprecision, measured as a coefficient of variation (CV) of MEA measurements, was determined by assessing samples five times from healthy subjects (controls) and from ACS patients on dual antiplatelet therapy. The mean CV of ADP MEA measurement was $3.9 \%$ in controls and $5.8 \%$ in ACS patients.

Genotyping was performed at the Green Cross Reference Laboratory in Korea. The base numbering and allele definitions followed the nomenclature of the $\mathrm{Hu}$ man CYP Allele Nomenclature Committee, and the CY$\mathrm{P}_{2} \mathrm{C}_{1} 9$ genotypes for $\mathrm{CrP}_{2} \mathrm{C}_{1} 9^{\star} 2$ (rs4244285, c. $681 \mathrm{G}>\mathrm{A}$, p. $\mathrm{P}_{22} \mathrm{P}$ ) and $\mathrm{CrP}_{2} \mathrm{C}_{19}{ }^{*} 3$ (rs4986893, c. 636G>A, p. W212X) were determined using the polymerase chain reaction (PCR)-SNaPshot method (Applied Biosystems, Foster City, CA, USA) using genomic DNA isolated from leukocytes of peripheral venous blood with an extraction kit (QIAamp ${ }^{\circledR}$ DNA Blood Mini Kit, Qiagen, Hilden, Germany). The genomic DNA region containing one of the two single nucleotide polymorphisms (SNPs) was amplified with PCR separately. The PCR product was processed as per the ABI SNaPshot protocol using primers designed for fluorescent dideoxy nucleotide termination. SNP analysis was carried out using the ABI 3100 genetic analyzer. In this study, $\mathrm{CrP}_{2} \mathrm{C}_{19}$ genotyping of HPR patients revealed three phenotypes: extensive metabolizers $\left(*_{1} / *_{1}\right)$, intermediate metabolizers $\left(*_{1} / *_{2}\right.$ and/or $\left.*_{1} / *_{3}\right)$, and poor metabolizers $\left({ }^{*} 2 / *^{*}, *_{2} / *^{*}\right.$, and/or $\left.{ }^{*} /{ }^{*} 3\right)$.

After blinded platelet function testing, patients were classified as responders or nonresponders. Nonresponder status has been specified based on a platelet aggregation value of $46.8 \mathrm{U}$ or greater [7]. We used $47 \mathrm{U}$ as the cutoff value for nonresponders, because platelet reactivity measured using the MEA kit shows the resulting numerical value as an integer. All the nonresponders underwent genotyping and repeated measurements of platelet reactivity after sequential maintenance doses of the four treatment strategies. Treatment strategies were $150 \mathrm{mg}$ daily clopidogrel, triple antiplatelet therapy with clopidogrel $75 \mathrm{mg}$ once and cilostazol $100 \mathrm{mg}$ twice daily, ticlopidine (Clid ${ }^{\circledR}$, Yuyu Pharmaceutical Inc., Seoul, Korea) $250 \mathrm{mg}$ twice daily, or ticagrelor $90 \mathrm{mg}$ twice daily, each lasting approximately $28 \pm 4$ days. All responders were included in the primary analysis without genotyping. At the end of each treatment period, ischemia, bleeding, and other adverse events were ascertained. After the last study drug treatment period, patients were recommended to follow the treatment strategy showing the best results.

This study was conducted in a manner consistent with ethical principles based on the Declaration of Helsinki. Informed consent was obtained from all participants. This study was approved by the Institutional Review Board of each participating hospital. 


\section{End points}

The primary endpoint was platelet reactivity during the study drug administration (platelet reactivity index [PRI]). PRI was obtained at each site with encrypted point-of-care, MEA, and reported as an arbitrary unit (U). Patients suffering from fatal cardiovascular or cerebrovascular events who needed medical treatment or admission and those who met the criteria for bleeding requiring medical attention were assessed at each visit. Adverse events and serious adverse events were documented.

\section{Adherence}

Standard pill count was used to assess adherence. At each visit, the number of remaining pills in the containers was counted. Adherence was defined by the number of pills taken (dispensed pills-returned pills) relative to the theoretical number of prescribed doses.

\section{Statistical analysis}

Continuous data were represented as mean \pm standard deviation (SD), and categorical data were represented as counts and percentages unless otherwise specified. The analytic dataset consisted of all patients who had successfully completed platelet function testing. No imputation was applied for missing data, and platelet function data were analyzed per protocol. Comparisons of baseline characteristics between responders and nonresponders were done using Student $t$ test or Pearson chi-square test as appropriate. Comparisons of baseline characteristics among responders and nonresponders with the four genotypes were done using either one-way analysis of variance (ANOVA) followed by Dunnett's multiple-comparison test if significant differences were detected, or Pearson chi-square/Kolmogorov tests depending on the type of variable. Association of the different treatment strategies with platelet reactivity among the nonresponders (with heterozygotes and homozygotes tested separately and combined) and responders was evaluated by using a repeated mixed model with patient response as a variable effect and treatment strategy as a fixed effect. Least square means (LSMs) and differences between means were calculated. A linear mixed model was also used to test equality of differences in LSMs among the treatment strategies in each genotype with patient response as a variable effect and treatment strategy as a fixed effect, followed by the Tukey-Kramer test to compare LSM of each treatment strategy in responders after $75 \mathrm{mg}$ daily clopidogrel intake. Differences in the LSMs were calculated and reported with 95\% confidence intervals (CIs). Proportions of nonresponders in each treatment strategy were compared using the Bonferroni test. Multiple logistic regression analysis was performed to determine independent risk factors for distinguishing responders from nonresponders with the $\mathrm{CrP}_{2} \mathrm{C}_{19} *_{1} / *_{1}$ genotype. Comparisons of clinical events between the responder group and each of the nonresponder group $\mathrm{s}$ were done using either the Student $t$ test or ANOVA as appropriate.

To attain at least $80 \%$ power to detect a $13.7 \mathrm{U}$ difference in the paired $U$ means of the treatment strategies with ADP-induced platelet aggregation (which corresponds to the observed difference seen in a similar study that compared the results of $20 \mu \mathrm{M}$ ADP-induced platelet aggregation with $75 \mathrm{mg}$ clopidogrel or ticlopidine in homozygous nonresponders) [8], and maintaining an overall $\alpha$ of 0.0125 for the study, at least 29 homozygous patients were required. In this calculation, the standard deviation of the difference was assumed to be $20 \%$. Based on an estimate of the proportion of Korean patients carrying mutant alleles of CYP $\mathrm{C}_{1} 9 *_{1} / *_{1}(50 \%)$, $\mathrm{CrP}_{2} \mathrm{C}_{19} *_{1} / *_{2}$ and $*_{1} / *_{3}(36.5 \%)$, and $\mathrm{CrP}_{2} \mathrm{C}_{1}{ }^{*} 2 / *_{2}$, $*_{2} / *_{3}$, and $*_{3} / *_{3}(13.5 \%)$ [9], the total sample sizes needed were at least 108, 75, and 29, respectively. We enrolled $30 \%$ more patients than the calculated sample size considering follow-up loss, and because we did not know the exact proportion of nonresponders with the mutant allele $\mathrm{CrP}_{2} \mathrm{C}_{1}{ }^{*}{ }_{1} /{ }^{*} 1$, we enrolled an additional $30 \%$ patients with this genotype. All statistical analyses were performed with the software SAS version 9.2 (SAS Inc., Cary, NC, USA). All analyses were two-tailed, and a p value of less than 0.05 was considered as the threshold for statistical significance.

\section{RESULTS}

In total, 376 patients were screened, and blood samples were obtained from all patients for blinded platelet function testing to identify response to clopidogrel. Their mean \pm SD age was 64.8 years, $61.7 \%$ were male, and $33.5 \%$ had a history of myocardial infarction. A total of 193 pa- 
Table 1. Patient baseline characteristics

\begin{tabular}{|c|c|c|c|c|}
\hline Characteristic & Overall $(\mathrm{n}=373)$ & Responders $(\mathrm{n}=193)$ & Nonresponders $(\mathrm{n}=183)$ & $p$ value \\
\hline Age, yr & $64.8 \pm 11.6$ & $63.8 \pm 11.5$ & $66.1 \pm 11.9$ & 0.0671 \\
\hline Male sex & $232(61.7)$ & $122(63.2)$ & $110(60.1)$ & 0.5361 \\
\hline Weight, kg & $162.4 \pm 8.1$ & $162.7 \pm 7.7$ & $162.1 \pm 8.6$ & 0.5389 \\
\hline Height, $\mathrm{cm}$ & $67.5 \pm 15.9$ & $66.8 \pm 16.5$ & $68.1 \pm 15.2$ & 0.4212 \\
\hline Body mass index ${ }^{\mathrm{a}}, \mathrm{kg} / \mathrm{m}^{2}$ & $25.1 \pm 3.7$ & $24 \cdot 6 \pm 3 \cdot 3$ & $25.7 \pm 4.0$ & 0.0046 \\
\hline $\mathrm{SBP}, \mathrm{mmHg}$ & $127 \cdot 4 \pm 18.6$ & $127.6 \pm 19.1$ & $127.2 \pm 18.3$ & 0.8257 \\
\hline $\mathrm{DBP}, \mathrm{mmHg}$ & $77.8 \pm 11.2$ & $78.9 \pm 11.1$ & $76.7 \pm 11.3$ & 0.0505 \\
\hline Heart rate, /min & $74.5 \pm 12.3$ & $74 \cdot 5 \pm 11.6$ & $74.4 \pm 12.9$ & 0.9356 \\
\hline Hypertension & $255(67.8)$ & $134(69.4)$ & $121(66.1)$ & 0.4923 \\
\hline Diabetes mellitus & $131(34.8)$ & $61(31.6)$ & $70(38.3)$ & 0.1765 \\
\hline Hyperlipidemia & $252(67.6)$ & $122(63 \cdot 5)$ & $130(71.0)$ & 0.1222 \\
\hline Family history & $25(6.7)$ & $13(6.7)$ & $14(7 \cdot 7)$ & 0.7314 \\
\hline History of MI & $125(33.5)$ & $65(33.7)$ & $63(34.4)$ & 0.8785 \\
\hline History of PCI & $312(83.6)$ & $168(87.1)$ & $144(78.7)$ & 0.0311 \\
\hline History of CABG & $36(9.7)$ & $14(7 \cdot 3)$ & $22(12.0)$ & 0.1163 \\
\hline
\end{tabular}

Values are presented as mean $\pm \mathrm{SD}$ or number $(\%)$.

SBP, systolic blood pressure; DBP, diastolic blood pressure; MI, myocardial infarction; PCI, percutaneous coronary intervention; CABG, coronary artery bypass graft.

${ }^{a}$ Calculated as weight in kilograms divided by height in meters squared.

Table 2. Patient baseline characteristics on phenotypes

\begin{tabular}{|c|c|c|c|c|c|}
\hline \multirow{2}{*}{ Characteristic } & \multirow{2}{*}{ Responder $(\mathrm{n}=193)$} & \multicolumn{3}{|c|}{ Non-responder $(n=183)$} & \multirow{2}{*}{$p$ value $^{a}$} \\
\hline & & $\operatorname{EM}(n=49)$ & $\operatorname{IM}(\mathrm{n}=97)$ & $\mathrm{PM}(\mathrm{n}=37)$ & \\
\hline Age, yr & $66.8 \pm 16.5$ & $71.8^{\mathrm{b}} \pm 8.8$ & $64.05 \pm 11.1$ & $66.4 \pm 13.9$ & $<0.0001$ \\
\hline Male sex & $122(63.2)$ & $31(63.3)$ & $56(56.5)$ & $23(62.2)$ & 0.8120 \\
\hline Weight, kg & $162.7 \pm 7.7$ & $161.6 \pm 7.4$ & $161.7 \pm 7.9$ & $162.6 \pm 10.4$ & 0.6144 \\
\hline Height, $\mathrm{cm}$ & $66.8 \pm 16.5$ & $73.7^{\mathrm{b}} \pm 15.4$ & $64.9 \pm 13.8$ & $66.3 \pm 13.2$ & 0.0221 \\
\hline Body mass index ${ }^{\mathrm{c}}, \mathrm{kg} / \mathrm{m}^{2}$ & $24.6 \pm 3 \cdot 3$ & $28.4^{b} \pm 4.2$ & $24 \cdot 4 \pm 3 \cdot 5$ & $25.1 \pm 3.4$ & $<0.0001$ \\
\hline $\mathrm{SBP}, \mathrm{mmHg}$ & $127.6 \pm 19.1$ & $131.8 \pm 19.9$ & $127.9 \pm 19.6$ & $121.2 \pm 14.6$ & 0.2311 \\
\hline $\mathrm{DBP}, \mathrm{mmHg}$ & $78.9 \pm 11.1$ & $78.0 \pm 12.5$ & $77.8 \pm 11.1$ & $71.0 \pm 9.5$ & 0.0021 \\
\hline Heart rate, $/ \mathrm{min}$ & $74.5 \pm 11.6$ & $76.0 \pm 13.3$ & $73.1 \pm 12.9$ & $73.9 \pm 13.9$ & 0.7123 \\
\hline Hypertension & $134(69.4)$ & $35(71.4)$ & $64(64 \cdot 5)$ & $22(59.5)$ & 0.6932 \\
\hline Diabetes mellitus & $61(31.6)$ & $22(44.9)$ & $34(34.8)$ & $14(37.8)$ & 0.5193 \\
\hline Hyperlipidemia & $122(63.5)$ & $39(79.6)$ & $66(71.9)$ & $25(67.6)$ & 0.2034 \\
\hline Family history of CAD & $13(6.7)$ & $1(2.0)$ & $4(2.8)$ & $9(24 \cdot 3)$ & 0.0032 \\
\hline History of MI & $65(33.7)$ & $11(22.5)$ & $35(36.8)$ & $17(46.0)$ & 0.3012 \\
\hline History of PCI & $168(87.1)$ & $37(75 \cdot 5)$ & $82(84.4)$ & $25(67.6)$ & 0.0394 \\
\hline History of CABG & $14(7 \cdot 3)$ & $5(10.2)$ & $12(15.8)$ & $5(13 \cdot 5)$ & 0.1467 \\
\hline
\end{tabular}

Values are presented as mean \pm SD or number $(\%)$.

EM, extensive metabolizers; IM, intermediate metabolizers: PM, poor metabolizers; SBP, systolic blood pressure; DBP, diastolic blood pressure; CAD, coronary artery disease; MI, myocardial infarction; PCI, percutaneous coronary intervention; CABG, coronary artery bypass graft.

${ }^{a} p$ values are calculated by analysis of variance, Pearson chi-square test, or Fisher exact test as appropriate.

b $p<0.05$, Dunnett's test compared with responder group.

${ }^{\mathrm{c} C a l c u l a t e d}$ as weight in kilograms divided by height in meters squared. 
tients were responders, whereas 183 were nonresponders (49 extensive metabolizers, $\mathrm{CrP}_{2} \mathrm{C}_{1} 9^{*} 1 /{ }^{*} 1 ; 97$ intermediate metabolizers, $71 \mathrm{CrP}_{2} \mathrm{C}_{1}{ }^{*}{ }_{1} / *_{2}$ and $26 \mathrm{CrP}_{2} \mathrm{C}_{1}{ }^{*} 1 /{ }^{*} 3$; and 37 poor metabolizers, $\mathrm{CrP}_{2} \mathrm{C}_{19}{ }^{*} 2$ and/or * ${ }_{3}$ ). Clinical characteristics did not differ between the responder and nonresponder groups (Tables 1 and 2). Adherence rates for the treatment strategies $150 \mathrm{mg}$ daily clopidogrel, triple therapy, ticlopidine $250 \mathrm{mg}$ twice daily, or ticagrelor $90 \mathrm{mg}$ twice daily among nonresponders on the study drug were $97.6 \%, 98.2 \%, 98.7 \%$, and $98.1 \%$, respectively.

\section{Response to interventions}

When treated with a standard clopidogrel maintenance dose of $75 \mathrm{mg}$ daily, platelet reactivity values in nonresponders were higher than those in responders on average (mean ADP test, 71.4 U, 95\% CI, 68.6 to 74.3; and 27.5 $\mathrm{U}, 95 \% \mathrm{CI}, 26.0$ to 28.9 , respectively; $p<0.001)$; this observation held true in all three phenotypes $(65.7 \mathrm{U}, 95 \%$ CI, 60.9 to 70.5 in extensive metabolizers; $70.8 \mathrm{U}, 95 \%$ CI, 64.8 to 76.9 in intermediate metabolizers; and 81.2 U, 95\% CI, 74.1 to 88.2 in poor metabolizers; $p<0.001$ for all comparisons). Each treatment strategy resulted in an approximate $22.3 \%$ to $63.4 \%$ absolute reduction in platelet reactivity, with some but not statistically significant reversal between the first two strategies. Among nonresponders, interventions with $150 \mathrm{mg}$ clopidogrel, triple therapy, ticlopidine, or ticagrelor produced significant reductions in platelet reactivity on average (55.5 U,
95\% CI, 52.4 to $58.7 ; 58.0 \mathrm{U}, 95 \%$ CI, 54.6 to $61.3 ; 32.8 \mathrm{U}$, 95\% CI, 30.2 to 35.4 ; and $26.1 \mathrm{U}, 95 \% \mathrm{CI}, 24.1$ to 28.1 ; $p$ $<0.001$ for all comparisons); this observation also held true in extensive metabolizers (49.2 U, $95 \% \mathrm{CI}, 44.7$ to 53.8; $55.4 \mathrm{U}, 95 \% \mathrm{CI}, 49.0$ to $61.8 ; 37.9 \mathrm{U}, 95 \% \mathrm{CI}, 33.1$ to 42.6; and 31.9 U, 95\% CI, 28.7 to 35.1; $p<0.001$ ), in intermediate metabolizers (54.1 U, 95\% CI, 46.9 to $61.2 ; 56.7$ U, $95 \%$ CI, 48.6 to $64.8 ; 30.0 \mathrm{U}, 95 \%$ CI, 24.7 to 37.5 ; and 24.0 U, 95\% CI, 19.6 to 28.4 ; $p<0.001$ ), and in poor metabolizers (69.5 U, 95\% CI, 61.5 to 77.5; $66.6 \mathrm{U}, 95 \% \mathrm{CI}$, 58.2 to 75.0 ; $30.3 \mathrm{U}, 95 \% \mathrm{CI}, 24.5$ to 36.0 ; and $23.2 \mathrm{U}, 95 \%$ CI, 18.0 to 28 ; $p<0.001$ ) (Table 3, Fig. 2). When we compared only platelet reactivity between responders and nonresponders with or without LOF alleles, ticagrelor similarly produced significant reductions in platelet reactivity on average (Table 4). For nonresponders on $75 \mathrm{mg}$ clopidogrel, proportions of nonresponders after each treatment were significantly reduced to $64.4 \%$ (116/180) with $150 \mathrm{mg}$ clopidogrel, to 68.4\% (121/177) with triple therapy, to $22.9 \%$ (25/175) with ticlopidine, and to $8.0 \%(24 / 168)$ with ticagrelor treatment. The risk ratios for nonresponder status with $150 \mathrm{mg}$ daily clopidogrel, triple therapy, ticlopidine, or ticagrelor were $0.64(95 \%$ CI, 0.58 to 0.72 ), 0.68 (95\% CI, 0.62 to 0.76 ), 0.23 (95\% CI, 0.17 to 0.30 ), and 0.08 ( $95 \% \mathrm{CI}, 0.05$ to 0.14 ), respectively. Proportions of nonresponders after the four above interventions, respectively based on genotype were $53.1 \%$, 60.4\%, 27.7\%, and 8.9\% in $\mathrm{CrP}_{2} \mathrm{C}_{1}{ }^{*} 1 /{ }^{*} 1 ; 64.3 \%, 69.6 \%$,

Table 3. On-treatment platelet reactivity

\begin{tabular}{|c|c|c|c|c|c|c|c|}
\hline & $\begin{array}{c}\text { Before } \\
\text { clopidogrel }\end{array}$ & $\begin{array}{c}75 \mathrm{mg} \\
\text { Clopidogrel }\end{array}$ & $\begin{array}{c}150 \mathrm{mg} \\
\text { Clopidogrel }\end{array}$ & Triple & Ticlopidine & Ticagrelor & $p$ value ${ }^{a}$ \\
\hline Responder & $76.3(72.8-79.8)$ & $27.5(26.0-28.9)$ & & & & & $<0.001$ \\
\hline No. of patients & 193 & 193 & & & & & \\
\hline Nonresponders & $90.2(86.6-93.9)$ & $71.4(68.6-74.3)$ & $55.5(52.4-58.7)$ & $58.0(54.6-61.3)$ & $32.8(30.2-35.4)$ & $26.1(24.1-28.0)$ & $<0.001$ \\
\hline No. of patients & 183 & 183 & 45 & 46 & 46 & 46 & \\
\hline EM & $72.9(67.5-78.4)$ & $65.7(60.9-70.5)$ & $49.2(44.7-53.8)$ & $55.4(49.0-61.8)$ & $37.9(33.1-42.6)$ & $31.9(28.7-35.1)$ & $<0.001$ \\
\hline No. of patients & 49 & 49 & 12 & 13 & 11 & 12 & \\
\hline IM & $97.9(88.6-103.2)$ & $70.8(64.8-76.9)$ & $54.1(46.9-61.2)$ & $56.7(48.6-64.8)$ & $30.0(24.7-37.5)$ & $24.0(19.6-28.4)$ & $<0.001$ \\
\hline No. of patients & 97 & 97 & 23 & 24 & 24 & 24 & \\
\hline $\mathrm{PM}$ & $108.4(94.8-112.1)$ & & $69.5(61.5-77.5)$ & $66.6(58.2-75.0)$ & $30.3(24.5-36.0)$ & $23.2(18.0-28.3)$ & $<0.001$ \\
\hline No. of patients & 37 & 37 & 10 & 9 & 11 & 10 & \\
\hline
\end{tabular}

Values are presented as mean (adenosine diphosphate-induced platelet reactivity index, 95\% confidence interval).

EM, extensive metabolizers; IM, intermediate metabolizers: PM, poor metabolizers.

${ }^{a} p$ value represents result of before clopidogrel versus the result after antiplatelet treatment. 

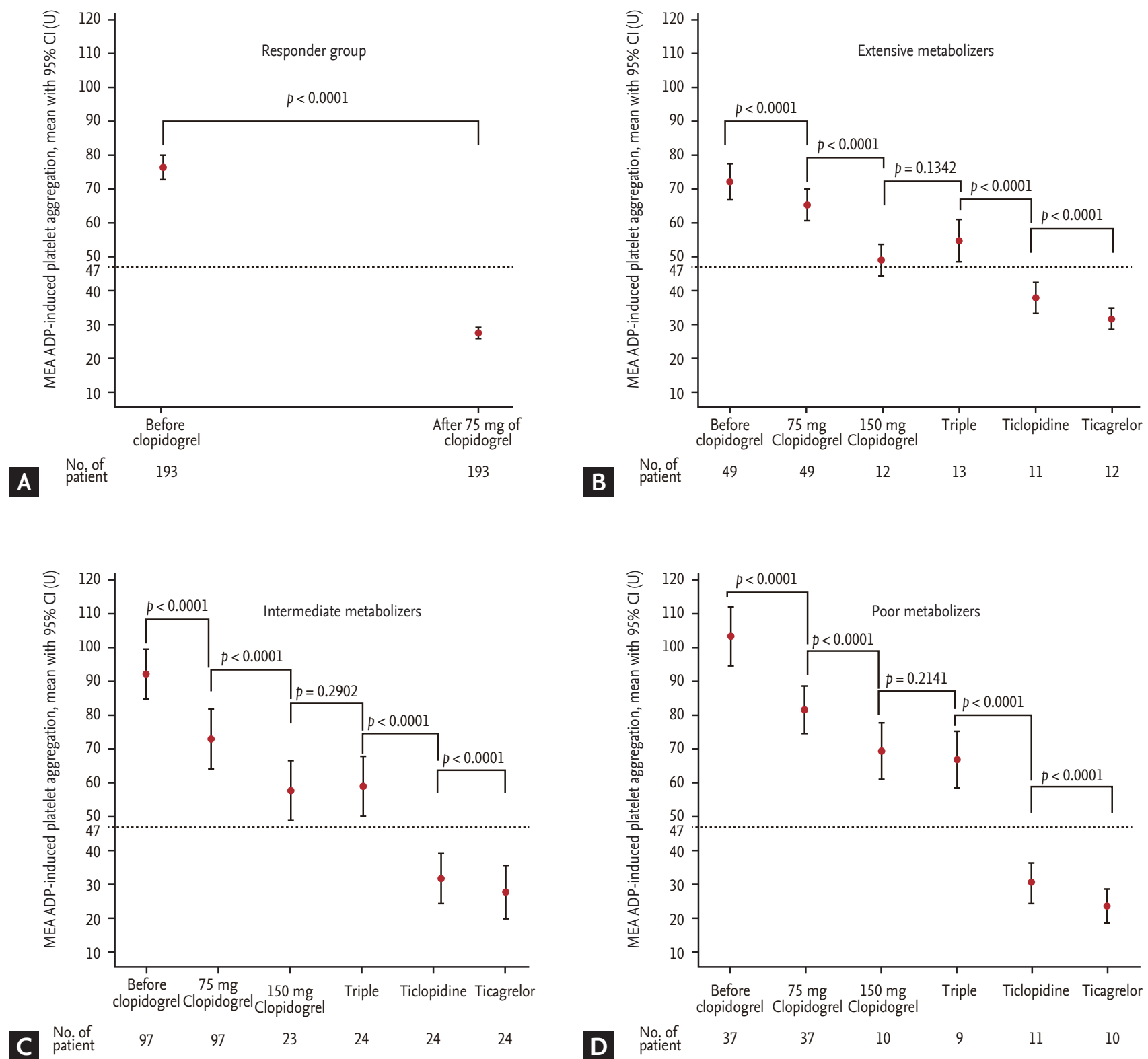

Figure 2. On-treatment platelet reactivity across phenotypes and treatments. Dotted line represents cutoff value of nonresponder. (A) Platelet reactivity on responder group. (B) Platelet reactivity on extensive metabolizers. (C) Platelet reactivity on intermediate metabolizers. (D) Platelet reactivity on poor metabolizers. MEA, multiple electrode aggregometry; ADP, adenosine diphosphate; CI, confidence interval.

$16.2 \%$, and $4.6 \%$ in $\mathrm{CYP}_{2} \mathrm{C}_{19}{ }^{*} 1 /{ }_{2} ; 56.0 \%, 60.0 \%, 24 \%$, and $13 \%$ in $\mathrm{CrP}_{2} \mathrm{C}_{1} 9{ }^{*} 1 /{ }^{*} 3$; and $86.1 \%, 82.9 \%, 28.6 \%$, and $11.8 \%$ in homozygotes (Fig. 3 ).

Among patients treated with $150 \mathrm{mg}$ daily clopidogrel or triple therapy, although the response to triple therapy was somewhat higher than that to $150 \mathrm{mg}$ daily clopidogrel without statistically significant differences $(p>0.05)$, the proportion of nonresponders and platelet reactivity in nonresponders with $75 \mathrm{mg}$ daily clopidogrel showed a significant reduction. However, the responses were still inferior to those seen with ticlopidine or ticagrelor treatment in all genotypes in nonresponders $(p<0.001$ for all). 
Table 4. On-treatment platelet reactivity (responder vs. nonresponder with or without LOF alleles)

\begin{tabular}{|c|c|c|c|c|c|c|c|}
\hline & $\begin{array}{c}\text { Before } \\
\text { clopidogrel }\end{array}$ & $\begin{array}{c}75 \mathrm{mg} \\
\text { Clopidogrel }\end{array}$ & $\begin{array}{c}150 \mathrm{mg} \\
\text { Clopidogrel }\end{array}$ & Triple & Ticlopidine & Ticagrelor & $p$ value $^{a}$ \\
\hline Responder & $76.3(72.8-79.8)$ & $27.5(26.0-28.9)$ & & & & & $<0.001$ \\
\hline No. of patients & 193 & 193 & & & & & \\
\hline Nonresponders & $90.2(86.6-93.9)$ & $71.4(68.6-74.3)$ & $55.5(52.4-58.7)$ & $58.0(54 \cdot 6-61.3)$ & $32.8(30.2-35.4)$ & $26.1(24.1-28.0)$ & $<0.001$ \\
\hline No. of patients & 183 & 183 & 45 & 46 & 46 & 46 & \\
\hline Without LOF alleles & $72.9(67.5-78.4)$ & $65.7(60.9-70.5)$ & $49.2(44.7-53.8)$ & $55.4(49.0-61.8)$ & $37.9(33.1-42.6)$ & $31.9(28.7-35.1)$ & $<0.001$ \\
\hline No. of patients & 49 & 49 & 12 & 13 & 11 & 12 & \\
\hline With LOF alleles & $107.5(82.5-110.2)$ & $77.1(68.1-82.2)$ & $61.8(53.1-69.6)$ & $60.5(52.1-65.7)$ & $30.7(24.7-37.4)$ & $23.9(18.7-28.8)$ & $<0.001$ \\
\hline No. of patients & 134 & 134 & 33 & 33 & 36 & 34 & \\
\hline
\end{tabular}

Values are presented as mean (adenosine diphosphate-induced platelet reactivity index, 95\% confidence interval).

LOF, loss of function.

${ }^{a} p$ value represents results of before clopidogrel versus the result after antiplatelet treatment.

\section{Responses after interventions in nonresponders with extensive metabolizer and intermediate metab- olizer phenotypes vs. those in responders, on $75 \mathrm{mg}$ daily clopidogrel}

When compared with patients in the responder group treated with $75 \mathrm{mg}$ daily clopidogrel, patients in the nonresponder group treated with $150 \mathrm{mg}$ clopidogrel or triple therapy showed higher LSMs of platelet reactivity in all genotype ( $p<0.001$ for all comparisons). Interventions with ticlopidine or ticagrelor in nonresponders resulted in platelet reactivity that was as good as the response to standard clopidogrel dosing in responder patients with an intermediate metabolizer phenotype. Treatment with ticagrelor resulted in a reduction of platelet reactivity levels in nonresponders with standard clopidogrel dosing regardless of genotype, which was similar to the results with standard clopidogrel dosing in responders (Fig. 4).

\section{Clinical events}

There were no deaths, cerebrovascular events needing medical treatment or admission, or Thrombolysis In Myocardial Infarction major or minor bleeding events. Among responders taking $75 \mathrm{mg}$ clopidogrel, one patient experienced a cardiac ischemic event. No patients with bleeding events met the criteria for bleeding requiring medical attention. Among nonresponders, one patient experienced a cardiac ischemic event with $75 \mathrm{mg}$ clopidogrel. A bleeding event requiring medical attention occurred in one patient in the $150 \mathrm{mg}$ daily clopido- grel group, two patients in the triple therapy group, and two patients in the ticagrelor group. No adverse events or serious adverse events that met end points occurred at a frequency greater than $3 \%$ per treatment group, and there were no significant hematologic, gastrointestinal, or musculoskeletal disorders in nonresponders in the different intervention groups.

\section{DISCUSSION}

Pharmacodynamic studies have demonstrated wide interindividual variability in the platelet inhibitory response to clopidogrel [10], and an observational study linked poor pharmacodynamic responses to cardiovascular events with the standard dose of clopidogrel [11]. HPR is associated with an increased risk of adverse events after coronary artery stenting [2], with this risk specifically linked to the presence of the LOF alleles $C Y$ $\mathrm{P}_{2} \mathrm{C}_{19}{ }^{*} 2$ and $\mathrm{CrP}_{2} \mathrm{C}_{19}{ }^{*} 3[12,13]$.

Interindividual variability in platelet response to clopidogrel, in addition to genetic variants of enzymes in metabolic pathways, results in high on-clopidogrel platelet reactivity [14]. In addition, it has been reported that clinical factors (noncompliance, under-dosing, poor absorption, drug-drug interactions, diabetes mellitus, and increased body mass index) and cellular factors (expression of receptors for clopidogrel and platelet turnover) may influence platelet response to clopidogrel $[11,15]$. In this regard, we evaluated and analyzed all non- 

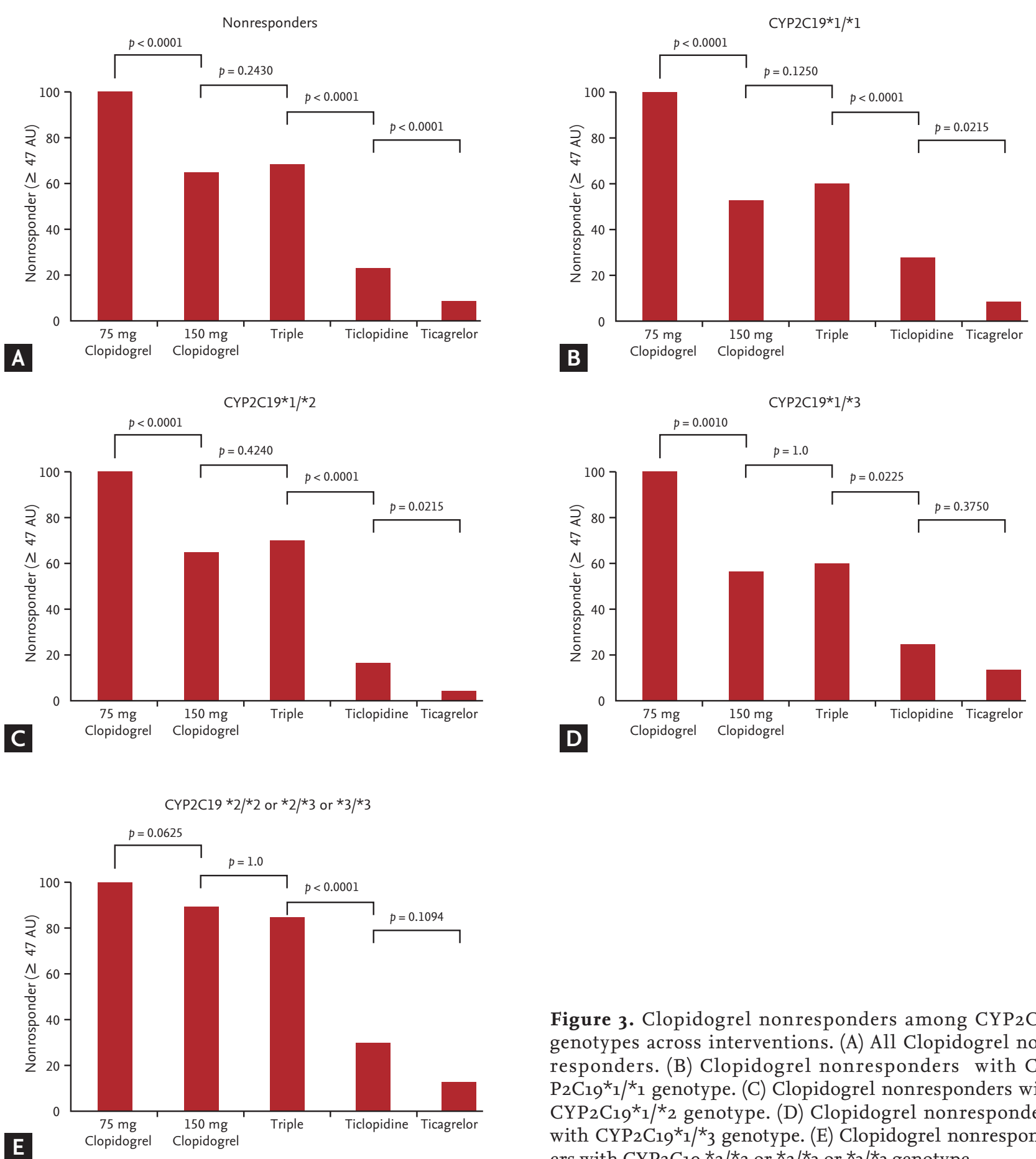

Figure 3. Clopidogrel nonresponders among $\mathrm{CYP}_{2} \mathrm{C}_{19}$ genotypes across interventions. (A) All Clopidogrel nonresponders. (B) Clopidogrel nonresponders with CY$\mathrm{P}_{2} \mathrm{C}_{19}{ }^{*} 1 /{ }^{*}$ genotype. (C) Clopidogrel nonresponders with $\mathrm{CYP}_{2} \mathrm{Cl}_{1}{ }^{*} 1 /{ }^{*} 2$ genotype. (D) Clopidogrel nonresponders with $\mathrm{CYP}_{2} \mathrm{C}_{1}{ }^{*} 1 / *_{3}$ genotype. (E) Clopidogrel nonresponders with $\mathrm{CYP}_{2} \mathrm{Cl}_{1} \star_{2} / \star_{2}$ or $\star_{2} / \star_{3}$ or $\star_{3} / \star_{3}$ genotype.

responders with or without the $\mathrm{CrP}_{2} \mathrm{C}_{19} \mathrm{LOF}$ alleles.

We found that the four interventions to reduce HPR with clopidogrel maintenance treatment resulted in more significant reductions in platelet reactivity in responders than in nonresponders with a standard dose of

75 mg clopidogrel. In nonresponders with $\mathrm{CrP}_{2} \mathrm{C}_{19} \mathrm{LOF}$ alleles, ticlopidine or ticagrelor resulted in similar platelet inhibition as in responders, but $150 \mathrm{mg}$ daily clopidogrel or triple therapy did not. Among all nonresponders with or without $\mathrm{CrP}_{2} \mathrm{C}_{19} \mathrm{LOF}$ alleles, only patients on 


\section{KJIM ${ }^{-}$}
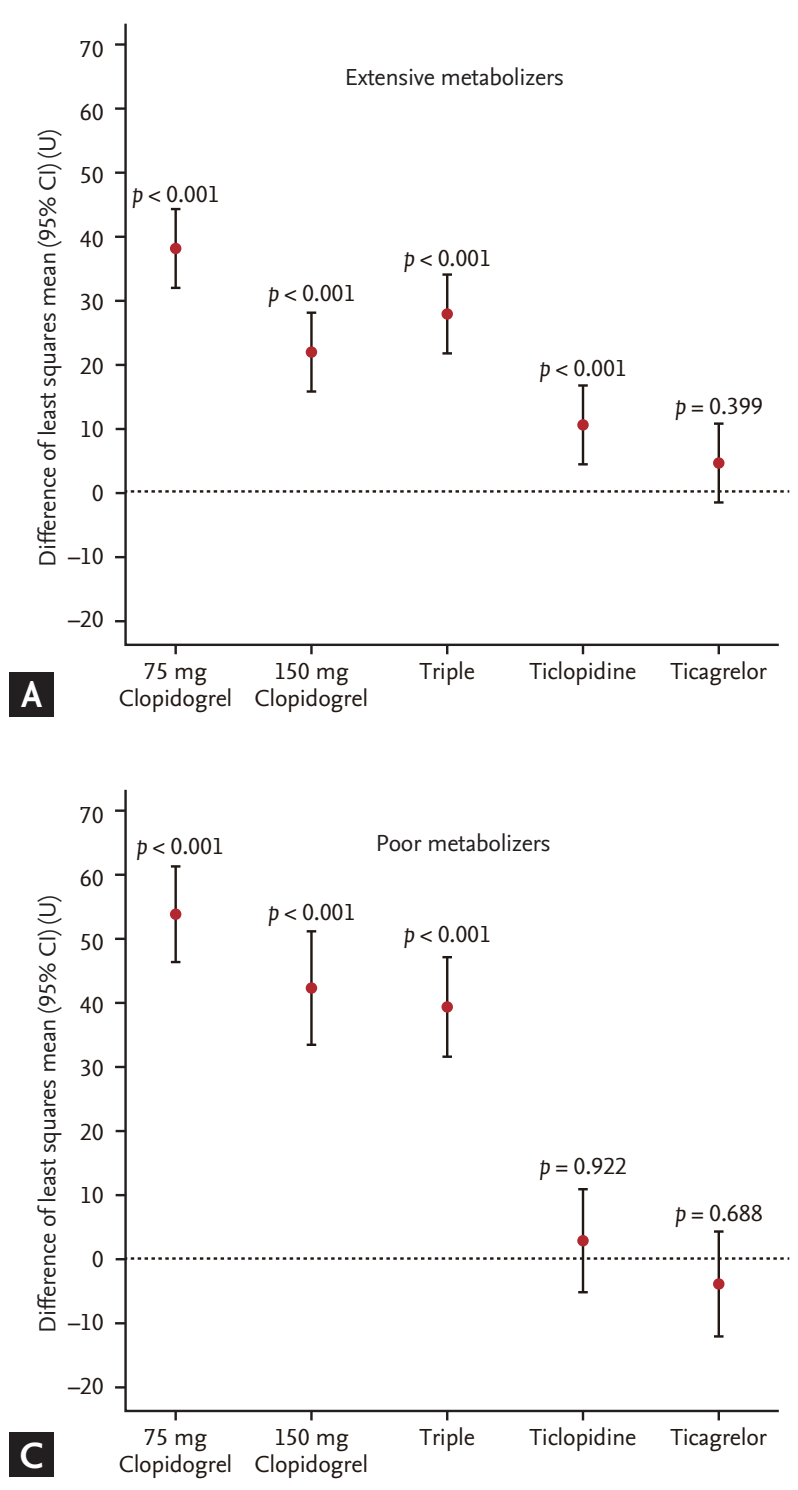

ticagrelor were able to achieve on-treatment platelet reactivity similar to that achieved with $75 \mathrm{mg}$ daily clopidogrel in responder patients with cardiovascular disease.

Several studies have evaluated methods of overcoming clopidogrel resistance. Different loading doses of clopidogrel (up to a total dose of $900 \mathrm{mg}$ ) based on the $\mathrm{CY}$ $\mathrm{P}_{2} \mathrm{C} 19$ genotype achieved a degree of platelet inhibition comparable with that observed in response to the standard $300 \mathrm{mg}$ loading dose in noncarrier patients [16]. However, high maintenance doses of clopidogrel (e.g., $150 \mathrm{mg}$ daily) did not show this degree of platelet inhibition [17]. Mega et al. [18] have reported that raising the daily dose of clopidogrel up to $225 \mathrm{mg}$ led to platelet inhibition in carriers of the $\mathrm{CrP}_{2} \mathrm{C}_{19} *_{2}$ alleles, similar to

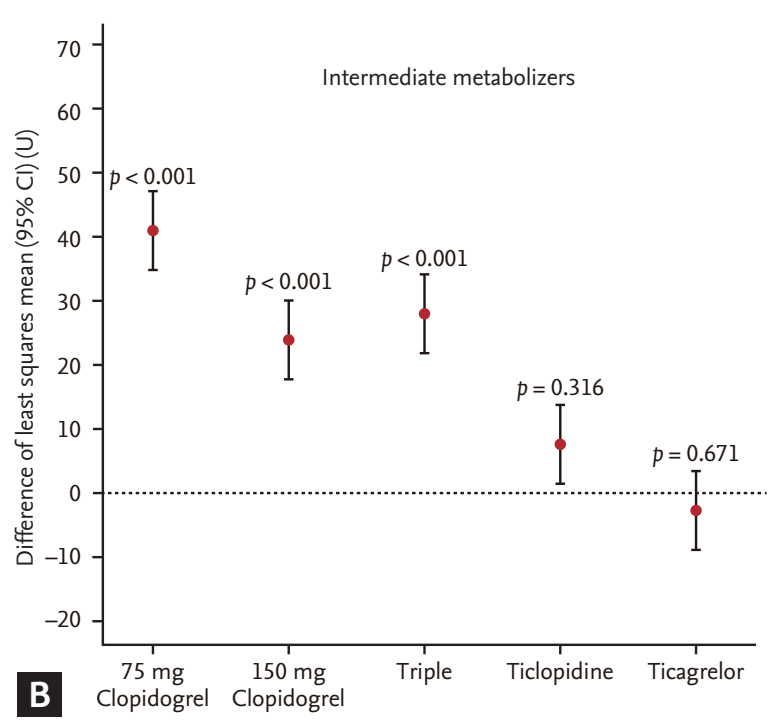

Figure 4. Difference in platelet reactivity between phenotypes treated with interventions versus responders treated with daily $75 \mathrm{mg}$ of clopidogrel on (A) extensive metabolizers, (B) intermediate metabolizers, and (C) poor metabolizers. Data are reported as least squares differences and $95 \%$ confidence intervals for platelet reactivity between nonresponders with phenotypes at clopidogrel doses of 75 $\mathrm{mg}, 150 \mathrm{mg}$, triple therapy, and ticlopidine, and ticagrelor and responders at $75 \mathrm{mg}$ of clopidogrel. Differences in least squares means were tested using Tukey-Kramer test. CI, confidence interval.

that seen in noncarriers except for homozygotes. In this report, increasing clopidogrel up to $300 \mathrm{mg}$ daily also did not show superior reduction of platelet inhibition in patients who were homozygotes (poor metabolizers) [18].

Our study also found that increasing the maintenance dose from 75 to $150 \mathrm{mg}$ in nonresponder patients did not (on average) inhibit platelet reactivity to the levels seen in responder patients taking $75 \mathrm{mg}$ clopidogrel.

Cilostazol, a phosphodiesterase type III inhibitor, has been shown to be a more effective inhibitor of platelet aggregation than $75 \mathrm{mg}$ daily clopidogrel in most studies. Cilostazol inhibits phosphodiesterase activity, suppresses cyclic adenosine monophosphate degradation, and activates vasodilation-stimulated protein 
phosphorylation. Thus, cilostazol acts on pathways downstream of clopidogrel action without involvement of the $\mathrm{P}_{2} \mathrm{Y}_{12}$ receptor $[9,19]$. Moreover, cilostazol is metabolized mainly by the $\mathrm{CYP}_{3} \mathrm{~A}$ pathway and, to a lesser extent, the $\mathrm{CrP}_{2} \mathrm{Cl}_{1} 9$ pathway, the major clopidogrel-metabolizing pathway [20].

Potential mechanisms contributing to the benefits of adjunctive cilostazol might include not only consistent platelet inhibition but also other pleiotropic effects beyond pure $\mathrm{P} 2 \mathrm{Y} 12$ receptor inhibition [21]. These pharmacologic aspects of cilostazol may explain why cilostazol is superior to dual antiplatelet therapy including a $75 \mathrm{mg}$ daily maintenance dose of clopidogrel.

One study revealed superior efficacy of cilostazol in the inhibition of platelet reactivity compared to highdose clopidogrel (e.g., $150 \mathrm{mg} /$ day) in patients with clopidogrel resistance (ACCEL-RESISTANCE study) [22].

On the other hand, there have been no reports showing platelet inhibition with cilostazol in nonresponders similar to that seen in responders or $\mathrm{CrP}_{2} \mathrm{C}_{19}$ noncarriers. A previous study reported that triple therapy did not show as much of a reduction in platelet reactivity as in noncarriers, although triple therapy significantly reduced on-treatment platelet reactivity compared with dual antiplatelet therapy [23]; however, a recent randomized clinical trial failed to show a significant clinical benefit of triple versus dual antiplatelet therapy [24].

We implemented a consecutive change in drug treatment from $150 \mathrm{mg}$ daily clopidogrel to triple therapy in the same group of nonresponders, regardless of the presence or absence of the $\mathrm{CYP}_{2} \mathrm{C}_{19}$ LOF allele. Triple therapy, although showing significant reduction in platelet inhibition compared with dual antiplatelet therapy, did not result in superior efficacy of platelet inhibition compared with high-dose clopidogrel (e.g., $150 \mathrm{mg} /$ day); further, it showed inferior results compared with $75 \mathrm{mg}$ clopidogrel in responders.

Another thienopyridine derivative, ticlopidine, exerts a protective effect against stent thrombosis and major adverse cardiac events [25]. Ticlopidine is also a prodrug metabolized by multiple CYPs, including $\mathrm{CrP}_{2} \mathrm{C}_{19}$ [26]. The pathways that activate ticlopidine have not been identified, but it is known that ticlopidine is not primarily metabolized through the $\mathrm{CrP}_{2} \mathrm{C}_{19}$ pathway which is the main clopidogrel-metabolic pathway [27]; however, ticlopidine is probably effective even in patients with
$\mathrm{CrP}_{2} \mathrm{C}_{19}$ polymorphism. A cross-over study compared poor responsiveness after taking clopidogrel or ticlopidine, and found that poor responsiveness to either clopidogrel or ticlopidine at steady state was common [28].

In one study, switching from clopidogrel to ticlopidine significantly decreased platelet aggregation in patients who were $\mathrm{CYP}_{2} \mathrm{C}_{19}$ homozygotes, similar to the results of the present study [8]. It has been reported that heightened platelet reactivity based on frequency of the $\mathrm{CrP}_{2} \mathrm{C}_{19} \mathrm{LOF}$ polymorphism is strikingly higher in Asians than in Caucasians [29].

In Koreans, it was reported that the proportion of patients carrying at least one $\mathrm{CYP}_{2} \mathrm{C}_{1} 9^{*} 2$ allele was $53 \%$, and that these patients had significantly higher clopidogrel on-treatment platelet reactivity than noncarriers [29]. Considering the presence of the $\mathrm{CrP}_{2} \mathrm{C}_{1} 9^{*} 3 \mathrm{LOF}$ allele, which is almost nonexistent in Caucasian patients, frequency of $\mathrm{CrP}_{2} \mathrm{C}_{19}$ LOF allele carriers exceeds $60 \%$ in the Korean population [30]. In the Japanese, the percentage of $\mathrm{CYP}_{2} \mathrm{C}_{1}$ * $_{2} \mathrm{LOF}$ carriers is reported to be approximately $42 \%$ [8].

Although its tolerability is poorer than that of clopidogrel $[25-27,29,30]$, ticlopidine may be a good choice for intervention in high on-clopidogrel reactivity in carriers of $\mathrm{CrP}_{2} \mathrm{C}_{19}$ loss-function polymorphisms, particularly in Asians if adequate blood tests are done during follow-up.

Prasugrel and ticagrelor are the newer $\mathrm{P} 2 \mathrm{Y} 12$ ADP receptor blockers that achieve significantly higher levels of platelet inhibition than clopidogrel [31]. In contrast to clopidogrel, the effects of prasugrel and ticagrelor are less affected by variants in the $\mathrm{CrP}_{2} \mathrm{C}_{19}$ gene (and correspondingly, no association between the $\mathrm{CrP}_{2} \mathrm{C}_{1} 9$ genotype and clinical events has been observed in patients treated with these agents) [32], and thus, these medications represent other alternative treatment strategies in such patients.

Ticagrelor is a new antiplatelet agent chemically known as a cyclopentyl triazolopyrimidine, with properties that distinguish it from the thienopyridines. Ticagrelor, irrespective of the $\mathrm{CrP}_{2} \mathrm{C}_{1}$ genotype also demonstrated superior pharmacodynamic effects compared with clopidogrel on inhibition of platelet reactivity [33]. In the present study, ticagrelor treatment showed similar platelet inhibition in responders and nonresponders with or without the LOF $\mathrm{CYP}_{2} \mathrm{C}_{19}$ alleles. 
There were several limitations in our analyses. First, we used only a platelet function test for the analysis. A prospective trial has established that HPR diagnosed using MEA is an independent risk factor for early stent thrombosis, whereas profound inhibition of platelet aggregation induced by ADP is an independent risk factor for bleeding. Moreover, the study assessed the predictive value of HPR in clopidogrel-treated patients and used the upper quintile of the AUC values in order to establish the cutoff MEA value [12]. Second, we focused on platelet reactivity as the primary outcome of interest. While platelet reactivity is a well-validated predictor of poor clinical outcomes, large-scale and long-term trials powered for clinical outcomes will be necessary to assess adverse events and establish more preferred treatment regimens. Third, because there is no reliable cutoff value of HPR in East Asian patients, we used Dr. Sibbing's cutoff HPR value.

In conclusion, this study demonstrates that among patients with stable angina, ticlopidine or ticagrelor treatment in nonresponder patients including all carriers of $\mathrm{CYP}_{2} \mathrm{Cl}_{1} *_{2}$ and/or * 3 alleles, compared with $150 \mathrm{mg}$ daily clopidogrel or triple therapy, achieved similar platelet reactivity as that in responders. In particular, ticagrelor resulted in a comparable degree of platelet inhibition in all nonresponders irrespective of genotype. These data help to define how patients with higher on-clopidogrel platelet reactivity and with different $\mathrm{CYP}_{2} \mathrm{C}_{1}$ genotypes respond to different evidence-based treatment strategies, and provide useful information to guide further clinical studies.

\section{KEY MESSAGE}

1. Post-percutaneous coronary intervention patients include clopidogrel nonresponders.

2. This study suggests that a combination of ticlopidine and ticagrelor is a good alternative to conventional maintenance therapy for clopidogrel nonresponders.

\section{Conflict of interest}

No potential conflict of interest relevant to this article was reported.

\section{REFERENCES}

1. Yusuf S, Zhao F, Mehta SR, et al. Effects of clopidogrel in addition to aspirin in patients with acute coronary syndromes without ST-segment elevation. N Engl J Med 2001;345:494-502.

2. Marcucci R, Gori AM, Paniccia R, et al. Cardiovascular death and nonfatal myocardial infarction in acute coronary syndrome patients receiving coronary stenting are predicted by residual platelet reactivity to ADP detected by a point-of-care assay: a 12-month follow-up. Circulation 2009;119:237-242.

3. Trenk D, Hochholzer W, Fromm MF, et al. Cytochrome $\mathrm{P}_{450}{ }_{2} \mathrm{C} 19$ 681G>A polymorphism and high on-clopidogrel platelet reactivity associated with adverse 1-year clinical outcome of elective percutaneous coronary intervention with drug-eluting or bare-metal stents. J Am Coll Cardiol 2008;51:1925-1934.

4. Shuldiner AR, O'Connell JR, Bliden KP, et al. Association of cytochrome $\mathrm{P}_{450} 2 \mathrm{C} 19$ genotype with the antiplatelet effect and clinical efficacy of clopidogrel therapy. JAMA 2009;302:849-857.

5. Mega JL, Simon T, Collet JP, et al. Reduced-function CY$\mathrm{P}_{2} \mathrm{C}_{1} 9$ genotype and risk of adverse clinical outcomes among patients treated with clopidogrel predominantly for PCI: a meta-analysis. JAMA 2010;304:1821-1830.

6. Toth O, Calatzis A, Penz S, Losonczy H, Siess W. Multiple electrode aggregometry: a new device to measure platelet aggregation in whole blood. Thromb Haemost 2006;96:781-788.

7. Sibbing D, Braun S, Morath T, et al. Platelet reactivity after clopidogrel treatment assessed with point-of-care analysis and early drug-eluting stent thrombosis. J Am Coll Cardiol 2009;53:849-856.

8. Maeda A, Ando H, Asai T, et al. Differential impacts of $\mathrm{CYP}_{2} \mathrm{C}_{19}$ gene polymorphisms on the antiplatelet effects of clopidogrel and ticlopidine. Clin Pharmacol Ther 2011;89:229-233.

9. Yoo HD, Cho HY, Lee YB. Population pharmacokinetic analysis of cilostazol in healthy subjects with genetic polymorphisms of $\mathrm{CYP}_{3} \mathrm{~A}_{5}, \mathrm{CYP}_{2} \mathrm{C}_{19}$ and $\mathrm{ABCB}_{1}$. Br J Clin Pharmacol 2010;69:27-37.

10. Bonello L, Tantry US, Marcucci R, et al. Consensus and future directions on the definition of high on-treatment platelet reactivity to adenosine diphosphate. J Am Coll Cardiol 2010;56:919-933. 
11. Angiolillo DJ, Fernandez-Ortiz A, Bernardo E, et al. Variability in individual responsiveness to clopidogrel: clinical implications, management, and future perspectives. J Am Coll Cardiol 2007;49:1505-1516.

12. Collet JP, Hulot JS, Pena A, et al. Cytochrome P450 2 C19 polymorphism in young patients treated with clopidogrel after myocardial infarction: a cohort study. Lancet 2009;373:309-317.

13. Mega JL, Close SL, Wiviott SD, et al. Cytochrome p-450 polymorphisms and response to clopidogrel. N Engl J Med 2009;360:354-362.

14. Gurbel PA, Bliden KP, Hiatt BL, O'Connor CM. Clopidogrel for coronary stenting: response variability, drug resistance, and the effect of pretreatment platelet reactivity. Circulation 2003;107:2908-2913.

15. Siasos G, Tousoulis D, Stefanadis C. Multiple mechanisms affect the clopidogrel response. J Am Coll Cardiol 2009;53:900-901.

16. Collet JP, Hulot JS, Anzaha G, et al. High doses of clopidogrel to overcome genetic resistance: the randomized crossover CLOVIS-2 (Clopidogrel and Response Variability Investigation Study 2). JACC Cardiovasc Interv 2011;4:392-402.

17. Price MJ, Berger PB, Teirstein PS, et al. Standard- vs highdose clopidogrel based on platelet function testing after percutaneous coronary intervention: the GRAVITAS randomized trial. JAMA 2011;305:1097-1105.

18. Mega JL, Hochholzer W, Frelinger AL 3rd, et al. Dosing clopidogrel based on $\mathrm{CYP}_{2} \mathrm{C}_{1} 9$ genotype and the effect on platelet reactivity in patients with stable cardiovascular disease. JAMA 2011;306:2221-2228.

19. Dindyal S, Kyriakides C. A review of cilostazol, a phosphodiesterase inhibitor, and its role in preventing both coronary and peripheral arterial restenosis following endovascular therapy. Recent Pat Cardiovasc Drug Discov 2009;4:6-14.

20. Hattori Y, Suzuki K, Tomizawa A, et al. Cilostazol inhibits cytokine-induced nuclear factor-kappaB activation via AMP-activated protein kinase activation in vascular endothelial cells. Cardiovasc Res 2009;81:133-139.

21. Ota H, Eto M, Ako J, et al. Sirolimus and everolimus induce endothelial cellular senescence via sirtuin 1 down-regulation: therapeutic implication of cilostazol after drug-eluting stent implantation. J Am Coll Cardiol 2009;53:2298-2305.

22. Jeong YH, Lee SW, Choi BR, et al. Randomized compar- ison of adjunctive cilostazol versus high maintenance dose clopidogrel in patients with high post-treatment platelet reactivity: results of the ACCEL-RESISTANCE (Adjunctive Cilostazol Versus High Maintenance Dose Clopidogrel in Patients With Clopidogrel Resistance) randomized study. J Am Coll Cardiol 2009;53:1101-1109.

23. Park KW, Park JJ, Lee SP, et al. Cilostazol attenuates on-treatment platelet reactivity in patients with $\mathrm{CYP}_{2} \mathrm{C}_{1} 9$ loss of function alleles receiving dual antiplatelet therapy: a genetic substudy of the CILON-T randomised controlled trial. Heart 2011;97:641-647.

24. Suh JW, Lee SP, Park KW, et al. Multicenter randomized trial evaluating the efficacy of cilostazol on ischemic vascular complications after drug-eluting stent implantation for coronary heart disease: results of the CILON-T (influence of CILostazol-based triple antiplatelet therapy ON ischemic complication after drug-eluting sten'T implantation) trial. J Am Coll Cardiol 2011;57:280-289.

25. Taniuchi M, Kurz HI, Lasala JM. Randomized comparison of ticlopidine and clopidogrel after intracoronary stent implantation in a broad patient population. Circulation 2001;104:539-543.

26. Hagihara K, Nishiya Y, Kurihara A, Kazui M, Farid NA, Ikeda T. Comparison of human cytochrome $\mathrm{P}_{450}$ inhibition by the thienopyridines prasugrel, clopidogrel, and ticlopidine. Drug Metab Pharmacokinet 2008;23:412-420.

27. Quinn MJ, Fitzgerald DJ. Ticlopidine and clopidogrel. Circulation 1999;100:1667-1672.

28. Campo G, Valgimigli M, Gemmati D, et al. Poor responsiveness to clopidogrel: drug-specific or class-effect mechanism? Evidence from a clopidogrel-to-ticlopidine crossover study. J Am Coll Cardiol 2007;50:1132-1137.

29. Oh IY, Park KW, Kang SH, et al. Association of cytochrome $\mathrm{P}_{450} 2 \mathrm{C}_{19}{ }^{*} 2$ polymorphism with clopidogrel response variability and cardiovascular events in Koreans treated with drug-eluting stents. Heart 2012;98:139-144.

30. Jeong YH, Tantry US, Kim IS, et al. Effect of $\mathrm{CYP}_{2} \mathrm{C}_{1}{ }^{*}{ }_{2}$ and $\star_{3}$ loss-of-function alleles on platelet reactivity and adverse clinical events in East Asian acute myocardial infarction survivors treated with clopidogrel and aspirin. Circ Cardiovasc Interv 2011;4:585-594.

31. Wiviott SD, Trenk D, Frelinger AL, et al. Prasugrel compared with high loading- and maintenance-dose clopidogrel in patients with planned percutaneous coronary intervention: the Prasugrel in Comparison to Clopidogrel for Inhibition of Platelet Activation and Aggrega- 
tion-Thrombolysis in Myocardial Infarction 44 trial. Circulation 2007;116:2923-2932.

32. Wallentin L, James S, Storey RF, et al. Effect of $\mathrm{CYP}_{2} \mathrm{Cl}_{19}$ and $\mathrm{ABCB} 1$ single nucleotide polymorphisms on outcomes of treatment with ticagrelor versus clopidogrel for acute coronary syndromes: a genetic substudy of the
PLATO trial. Lancet 2010;376:1320-1328.

33. Tantry US, Bliden KP, Wei C, et al. First analysis of the relation between $\mathrm{CYP}_{2} \mathrm{C}_{1}$ genotype and pharmacodynamics in patients treated with ticagrelor versus clopidogrel: the ONSET/OFFSET and RESPOND genotype studies. Circ Cardiovasc Genet 2010;3:556-566. 